\title{
Concentrated growth factor exudate enhances the proliferation of human periodontal ligament cells in the presence of TNF- $\alpha$
}

\author{
XIAOJU LI ${ }^{1}$, HUIXIAO YANG ${ }^{2}$, ZIJIAN ZHANG ${ }^{3}$, ZHONGHAI YAN $^{3}$, HULING LV $^{2}$, YAN ZHANG ${ }^{4}$ and BIN WU ${ }^{1}$ \\ ${ }^{1}$ Department of Stomatology, The People's Hospital of Longhua, Shenzhen, Guangdong 518109; \\ ${ }^{2}$ Key Laboratory of Oral Medicine, Guangzhou Institute of Oral Disease, Stomatological Hospital of \\ Guangzhou Medical University, Guangzhou, Guangdong 510140, P.R. China; ${ }^{3}$ Department of Biomedical Sciences, \\ Paul L. Foster School of Medicine, Texas Tech University Health Sciences Center El Paso, El Paso, TX 79905, USA; \\ ${ }^{4}$ Department of General Therapy Dentistry, Stomatology Hospital of Jilin University, Changchun, Jilin 130021, P.R. China
}

Received June 11, 2018; Accepted November 12, 2018

DOI: $10.3892 / \mathrm{mmr} .2018 .9714$

\begin{abstract}
The purpose of this study was to evaluate the effects of concentrated growth factor exudate (CGFe) on human periodontal ligament cells (hPDLCs) stimulated by tumor necrosis factor (TNF)- $\alpha$. From the peripheral blood of healthy donors, CGFe was prepared according to the Sacco protocol after 7 days of incubation. The hPDLCs were cultured by a tissue explant method and identified with anti-vimentin and anti-cytokeratin antibodies. Cells were subjected to four different treatments: i) Control; ii) TNF- $\alpha$ (10 ng/ml); iii) CGFe (concentration 50\%); and iv) CGFe+TNF- $\alpha$. The proliferation of hPDLCs was measured with Cell Counting Kit-8 assays. Osteogenic differentiation and mineralization were determined by Alizarin Red S staining, alkaline phosphatase activity, western blotting and reverse transcription-quantitative polymerase chain reaction. CGFe enhanced cell proliferation and upregulated ALP activity, the mineralization level, and osteogenic-associated osteocalcin, runt-related transcription factor 2 and Osterix gene expression in hPDLCs under inflammatory conditions induced by TNF- $\alpha$. The present study demonstrated that CGFe enhanced hPDLC proliferation and osteogenic differentiation in the presence of TNF- $\alpha$-induced inflammation. As CGFe can be obtained from the venous blood of patients, it generates no immune reaction. Thus, it is more economical and beneficial to use $\mathrm{CGFe}$ in clinical periodontal regeneration practice than synthetic growth factors.
\end{abstract}

Correspondence to: Dr Huixiao Yang, Key Laboratory of Oral Medicine, Guangzhou Institute of Oral Disease, Stomatological Hospital of Guangzhou Medical University, 59 Huangsha Road, Guangzhou, Guangdong 510140, P.R. China

E-mail: huixiao.yang@ttuhsc.edu

Key words: concentrated growth factor exudate, tumor necrosis factor- $\alpha$, human periodontal ligament cells, osteogenic differentiation

\section{Introduction}

Periodontitis and chronic apical periodontitis often cause periodontal tissue destruction and severe alveolar bone defects (1). In periodontally compromised teeth, when partial or full socket wall destruction is evident, connective tissue will grow into the extraction site and lead to a deficient ridge (1). Periodontitis is widely treated with instant implant technology in clinical practice (2). However, periodontal tissue destruction and alveolar bone defects significantly weaken the stability and shorten the service life of dental implants (2). To improve the success rate of dental implants, concentrated growth factor (CGF) can be used to promote periodontal tissue regeneration and osteogenic differentiation (3). CGF is a gel-like substance, obtained by centrifugation of venous blood, which is rich in growth factors and fibrin (4-8), and CGF combined with bone graft material promotes immediate periodontal tissue regeneration and osteogenic differentiation $(8,9)$.

Previous studies have reported that platelet-rich plasma (PRP) and platelet-rich fibrin (PRF) in the first and second phases promote tissue regeneration, inhibit infection, regulate inflammation and reduce postoperative reactions (9-14). CGF is a third-phase plasma extract, consisting of multiple growth factors (4-8). Due to the unique structure of CGF fibrin, it can replace the diaphragm used in guiding bone regeneration to a certain extent $(8,9,15)$. CGF has been widely studied in the fields of oral implantation, extraction site preservation, jaw cyst treatment, and fracture healing promotion $(4-8,16,17)$.

CGF exudate (CGFe) is extracted from CGF and used in research labs to study the effects of CGF. Previous studies have shown that CGFe significantly shortens the time for osteogenesis in the operational area and distinctly improves bone formation quality $(8,9)$. CGFe has been demonstrated to stimulate the proliferation of human periodontal ligament fibroblasts (PDLFs) (17). However, these previous studies were performed without the influence of inflammatory factors, and therefore did not sufficiently reflect the clinical environment. Particularly, the effect of CGFe on enhancing the proliferation of hPDLCs in the presence of tumor necrosis factor (TNF)- $\alpha$-induced inflammation has not yet been investigated, to the best of our knowledge. 
TNF- $\alpha$ is a pro-inflammatory cytokine that is a critical pathological factor for the development of apical inflammation, which significantly inhibits osteogenic differentiation $(16,18-21)$. As TNF- $\alpha$-induced inflammation is commonly encountered in clinical practice $(16,20)$, it is of great clinical interest to evaluate the effects of CGFe in the presence of TNF- $\alpha$.

In the present study, the effects of CGFe on hPDLC proliferation and osteogenic differentiation were investigated in an inflammatory environment, stimulated by TNF- $\alpha$. Specifically, the effects of CGFe on alkaline phosphatase (ALP) activity, mineralization, and osteocalcin $(O C N)$, runt-related transcription factor 2 (RUNX2), and Osterix (OSX) gene expression in hPDLCs under TNF- $\alpha$-induced inflammatory conditions were examined.

\section{Materials and methods}

Preparation of CGFe. The present study was approved by the Ethics Committee of the Jilin University Health Science Center (Changchun, China). In accordance with this committee, CGFe was obtained from three healthy male donors who had visited the outpatient clinic at the Health Center of Jilin University (Changchun, China) from August 2017 to March 2018. They were non-smokers and non-drinkers aged from 22 to 30 years old, and their informed consent was obtained. The experiments described below were carried out under similar conditions and procedures as the previous study (11).

Venous blood samples from the donors were used to produce human CGF according to a previously described protocol (22). In brief, blood samples were centrifuged at $750 \mathrm{x}$ g for $12 \mathrm{~min}$ at $4^{\circ} \mathrm{C}$. Between the acellular plasma and red blood cells (RBCs), a white CGF clot formed. The CGF clot was held with sterile forceps, separated from the RBCs using scissors, placed on the grid of an endo box and compressed by the endo box cover. After $1 \mathrm{~min}$ of applied pressure, the CGF clot was converted into CGF membrane, and the CGF exudate (CGFe) was collected in the tray of the endo box.

The CGFe was centrifuged at $500 \mathrm{x}$ g for $5 \mathrm{~min}$ at $4^{\circ} \mathrm{C}$ to remove RBCs. Then, the $\mathrm{CGFe}$ was precipitated and filtered using a $0.22 \mu \mathrm{m}$ sterile syringe filter unit (Merck KGaA, Darmstadt, Germany). The pooled CGFe samples were stored at $-80^{\circ} \mathrm{C}$ prior to use. The original concentration of $\mathrm{CGFe}$ was defined as $100 \%$, and a $50 \%$ concentration of $\mathrm{CGFe}$ was obtained by dilution with Minimal Essential Medium- $\alpha$ ( $\alpha$-MEM; Gibco; Thermo Fisher Scientific, Inc., Waltham, MA, USA) and used in subsequent experiments.

Human periodontal ligament cell ( $h P D L C)$ culture. In total, 10 healthy and noncarious premolars from three male and two female donors aged from 13 to 18 years old who had received orthodontic treatment at the Oral and Maxillofacial Surgery Department of the Stomatology School of Jilin University (Changchun, China) were obtained with informed consent. Periodontal ligaments were gently scraped from the middle third of the tooth-root surface with a sharp scalpel, minced with ophthalmic scissors and rinsed with $\alpha$-MEM. These explants were cultured in $\alpha$-MEM supplemented with $10 \%$ fetal bovine serum (FBS; GE Healthcare Life Sciences, Chicago, IL, USA), $1 \%$ streptomycin and penicillin (Gibco; Thermo Fisher
Scientific, Inc.) at $37^{\circ} \mathrm{C}$ in $5 \% \mathrm{CO}_{2}$. Examination by inverted light microscopy (IX73; Olympus Corporation, Tokyo, Japan) at magnifications of $\mathrm{x} 20$ or $\mathrm{x} 40$ was performed daily, and the medium was replaced every 3 days. Once cells reached $80 \%$ confluence, they were transferred to a $75 \mathrm{~cm}^{2}$ flask, and this was defined as passage one. The same procedure was performed repeatedly to produce multiple passages. Cells prior to passage 6 were used in the present study.

Immunocytochemistry staining. hPDLCs at passage 3 were seeded into several 24 -well plates at a density of $1 \times 10^{4} /$ well and covered in advance with circular coverslips (diameter, $14 \mathrm{~mm}$ ) and incubated for $48 \mathrm{~h}$ at $37^{\circ} \mathrm{C}$. Cells were then rinsed three times with $0.01 \mathrm{M}$ PBS and fixed with $4 \%$ paraformaldehyde for $20 \mathrm{~min}$ at room temperature. Following a further wash with PBS, $0.25 \%$ Triton X-100 was added into the 24-well plates, which were incubated at $37^{\circ} \mathrm{C}$ for $15 \mathrm{~min}$. Endogenous peroxidase activity was eliminated by incubation with $3 \% \mathrm{H}_{2} \mathrm{O}_{2}$ for $10 \mathrm{~min}$ at room temperature. Then, hPDLCs were incubated with $1 \%$ bovine serum albumin (Gibco; Thermo Fisher Scientific, Inc.) and $22.52 \mathrm{mg} / \mathrm{ml}$ glycine in PBS $+0.1 \%$ Tween-20 for $30 \mathrm{~min}$ at room temperature. Next, cells were incubated with anti-vimentin (1:100; cat. no. ab24525; Abcam, Cambridge, MA, USA) and anti-cytokeratin (1:200; cat. no. AM06387SU-N; OriGene Technologies, Inc., Beijing, China) primary antibodies overnight at $4{ }^{\circ} \mathrm{C}$. The SP immunohistochemistry assay kit (cat. no. SP9001; ZSGB-BIO; OriGene Technologies, Inc., Beijing, China) was used for immunocytochemical staining according to the manufacturer's instructions, and a 3,3'-diaminobenzidine was used to stain positive cells. An inverted microscope (IX73; Olympus Corporation, Tokyo, Japan) was used to view the cells at magnifications of x20 or x40.

Cell Counting Kit (CCK)-8 assay. The objective of this experiment was to examine the effect of CGFe on the proliferation of hPDLCs in vitro. CGFe was obtained according to a previous protocol (16). The CCK-8 assay (Dojindo Laboratories, Kumamoto, Japan) was used to determine the effects of CGFe on hPDLC proliferation.

hPDLCs $\left(2 \times 10^{3} /\right.$ well) were seeded into a 96-well plates containing complete medium ( $\alpha$-MEM supplemented with $10 \%$ FBS, $1 \%$ streptomycin and penicillin) with $10 \% \mathrm{FBS}$ and incubated for $24 \mathrm{~h}$ at $37^{\circ} \mathrm{C}$. Then, hPDLCs were exposed to CGFe (concentration $50 \%)$, TNF- $\alpha(10 \mathrm{ng} / \mathrm{ml})$, or CGFe+TNF- $\alpha$ for 24,48 or $72 \mathrm{~h}$ at $37^{\circ} \mathrm{C}$, respectively. There was one additional control group with no CGFe or TNF- $\alpha$ treatment (complete medium with $10 \%$ FBS only). Cell proliferation was determined using the CCK-8 kit at the specified time-points. Kit reagent $(10 \mu \mathrm{l})$ was added to the culture medium in each well. After a $90 \mathrm{~min}$ incubation at $37^{\circ} \mathrm{C}$, absorbance at $450 \mathrm{~nm}$ was detected using an automatic microplate reader (Infinite 200 PRO; Tecan Group Ltd., Mannedorf, Switzerland). A well containing complete medium and CCK-8 solution without seeding cells was used as a further control. The assay was performed in duplicate, and the experiments were repeated six times under the same conditions.

ALP activity assay. hPDLCs $\left(500 \mu \mathrm{l} ; 1 \times 10^{4} /\right.$ well $)$ were seeded into 24-well plates and incubated for $24 \mathrm{~h}$ at $37^{\circ} \mathrm{C}$. Next, 
the cells were exposed to CGFe, TNF- $\alpha$ or CGFe+TNF- $\alpha$ for 7 or 14 days. At the given time-points, cells were lysed with $0.1 \%$ Triton $\mathrm{X}-100$, and the lysates were centrifuged at $8000 \mathrm{x} \mathrm{g}$ for $10 \mathrm{~min}$ at $4^{\circ} \mathrm{C}$. The supernatant $(50 \mu \mathrm{l} /$ well $)$ was added to 96-well plates, and ALP activity was examined with a ALP assay kit (Nanjing Jiancheng Bioengineering Institute, Nanjing, China) according to the manufacturer's protocols. The optical density (OD) values were read at $520 \mathrm{~nm}$ with an automatic microplate reader (Infinite 200 PRO; Tecan Group, Ltd.).

Osteogenic differentiation induction and Alizarin Red $S$ staining. hPDLCs $\left(500 \mu \mathrm{l} ; 1 \times 10^{4} /\right.$ well $)$ were seeded into 24-well plates in standard medium ( $\alpha$-MEM supplemented with $10 \%$ FBS, $1 \%$ streptomycin and penicillin) until $60-70 \%$ confluence was reached. The medium was then replaced with four different media: i) Mineral induction medium (MM; $\alpha$-MEM medium containing $10 \% \mathrm{FBS}, 50 \mathrm{mg} / \mathrm{ml}$ ascorbic acid, $10 \mathrm{mM} \beta$-glycerolphosphate and $10^{-8} \mathrm{M}$ dexamethasone); ii) $\mathrm{MM}$ with $\mathrm{CGFe}$ (concentration $50 \%$ ); iii) $\mathrm{MM}$ with TNF- $\alpha$ (10 ng/ml); or iv) MM with CGFe+TNF- $\alpha$ (10 ng/ml). The medium was replaced every 3 days.

Alizarin Red staining was used to detect and quantify the formation of mineralized nodules after 21 days. This assay was performed according to a previously described protocol, with minor modifications $(9,23)$. Specifically, the hPDLCs were fixed with $95 \%$ ethanol for $15 \mathrm{~min}$ at room temperature, washed twice with $\mathrm{dH}_{2} \mathrm{O}$ and stained with $0.1 \%$ Alizarin Red S solution ( $\mathrm{pH} 4.1$ ) for $20 \mathrm{~min}$ at room temperature. hPDLCs were observed under an inverted phase-contrast microscope (IX73; Olympus Corporation, Tokyo, Japan; magnification, x20 or x40). Next, the hPDLCs were washed three times with $\mathrm{dH}_{2} \mathrm{O}$. To semi-quantify the content of mineralized matrix nodules generated from the hPDLCs, $100 \mathrm{mM}$ cetyl pyridinium chloride was added to the 24-well plates to dissolve and release the calcium-combined Alizarin Red S into solution. OD values were read at $570 \mathrm{~nm}$, which represented the relative quantity of mineralization nodules.

Western blotting. Cell lysates were prepared in radioimmunoprecipitation assay buffer $(150 \mathrm{mM} \mathrm{NaCl}, 0.1 \%$ SDS, $1 \mathrm{mM}$ PMSF, 10 mM Tris- $\mathrm{Cl}$, pH 7.4, $1 \%$ sodium deoxycholate, $1 \%$ Triton X-100). The cells were treated with four different media (control, CGFe, TNF- $\alpha$, or CGFe+TNF- $\alpha$ ) for 14 days. The cell lysates were incubated for $30 \mathrm{~min}$ on ice, then clarified by centrifugation at $6,000 \mathrm{x}$ g for $10 \mathrm{~min}$ at $4^{\circ} \mathrm{C}$. Protein concentration was determined with a bicinchoninic acid assay. Lysate samples $(20 \mu 1)$ were denatured and resolved by $10 \%$ SDS-PAGE, transferred onto polyvinylidene difluoride membranes and run at $300 \mathrm{~mA}$ for $2 \mathrm{~h}$. Membranes were blocked in 5\% non-fat milk at room temperature for $1 \mathrm{~h}$, then incubated with primary antibodies against RUNX2 (1:1,000; cat. no. 12556; Cell Signaling Technology, Inc., Danvers, MA, USA) and Osterix (1:1,000; cat. no. ab229258; Abcam) and GAPDH overnight at $4^{\circ} \mathrm{C}$. Membranes were subsequently washed three times in Tris-buffered saline with $0.1 \%$ Tween 20 (TBST) and incubated with horseradish peroxidase-conjugated goat anti-rabbit IgG (1:500; cat. no. 10285-1-AP; ProteinTech Group, Inc., Chicago, IL, USA) for $1 \mathrm{~h}$ at room temperature. Following three washes with TBST, the protein bands were visualized by using an Enhanced Chemiluminescence kit (GE Healthcare Life Sciences) and exposed to X-ray film. GAPDH was used as internal reference and the ImageJ (version 1.48; National Institutes of Health, Bethesda, MD, USA) was used for densitometry.

Reverse transcription-quantitative polymerase chain reaction $(R T-q P C R)$. hPDLCs $\left(2 \mathrm{ml} ; 1 \times 10^{5} /\right.$ well $)$ were seeded into 6 -well plates in standard medium until $60-70 \%$ confluence was reached. The cells were treated with four different media (control, CGFe, TNF- $\alpha$, or CGFe+TNF- $\alpha$ ). The control group was incubated with osteogenic induction medium [ $\alpha$-MEM, $150 \mu \mathrm{g} / \mathrm{ml}$ ascorbic acid and $10 \mathrm{mM} \beta$-sodium glycerophosphate (Sigma-Aldrich; Merck KGaA)]. The experimental groups were treated with osteogenic induction medium plus CGFe, osteogenic induction medium plus TNF- $\alpha(10 \mathrm{ng} / \mathrm{ml})$, or osteogenic induction medium plus CGFe+TNF- $\alpha$ (10 ng/ml). RNA was extracted and reverse transcribed into cDNA using TRIzol $^{\circledR}$ reagent (Invitrogen; Thermo Fisher Scientific, Inc.) after 4, 7 and 14 days. cDNA synthesis was performed with $1 \mu \mathrm{g}$ of total RNA, random hexamer primers, $5 \mathrm{X}$ First-Strand Buffer, and dNTP Mix (10 mM each) using SuperScript ${ }^{\text {TM }}$ II RT (cat. no. 18064014; Invitrogen; Thermo Fisher Scientific, Inc.). The temperature protocol for RT was: Denaturing RNA at $65^{\circ} \mathrm{C}$ for $5 \mathrm{~min}$, then incubation at $25^{\circ} \mathrm{C}$ for $10 \mathrm{~min}$ and $42^{\circ} \mathrm{C}$ for $60 \mathrm{~min}$, followed by $70^{\circ} \mathrm{C}$ for $15 \mathrm{~min}$.

A $10 \mu 1$ volume reaction system was adopted for qPCR determination of the expression of the osteogenic genes $O C N$, $R U N X 2$ and $O S X$ using the SYBR ${ }^{\circledR}$ Premix Ex Taq $^{\mathrm{TM}}$ II kit (Takara Bio, Inc., Otsu, Japan). qPCR primers were designed to span an intron so that only RNA-specific amplification was possible. PCR was performed with the following thermocycling conditions: $95^{\circ} \mathrm{C}$ for $3 \mathrm{~min}$, followed by 40 cycles of $95^{\circ} \mathrm{C}$ for $3 \mathrm{sec}$ and $60^{\circ} \mathrm{C}$ for $60 \mathrm{sec}$. Each sample was tested in triplicate, and fold differences in gene expression were calculated using the $2^{-\Delta \Delta \mathrm{Cq}}$ method (24) with normalization to $\beta$-actin. The primer sequences are presented in Table I.

Statistical analysis. hPDLC proliferation and ALP activity assays were analyzed by one-way analysis of variance (ANOVA) followed by Tukey's post-hoc test. Western blotting and Alizarin Red staining assay data were analyzed using two-way ANOVA, followed by Bonferroni's post-hoc comparisons test for independent samples. SPSS 17.0 (SPSS, Inc., Chicago, IL, USA) was used to perform statistical analysis. $\mathrm{P}<0.05$ was considered to indicate a statistically significant difference. All data were expressed as the mean \pm standard deviation, and experiments were performed in triplicate.

\section{Results}

Characterization of hPDLCs. hPDLCs grew out from the tissue explant after 7 and 10 days of culture (Fig. 1A). Spindle shapes were observed, and a number of cells were distributed in a circinate pattern with rapid proliferation (Fig. 1B). The CGFe obtained was a yellowish clear fluid, and each $50 \mathrm{ml}$ blood sample produced $4 \mathrm{ml}$ of CGFe. The cells were vimentin positive (Fig. 1C) and keratin negative (Fig. 1D) according to immunochemistry staining, indicating that these primary cells were of mesenchymal origin. 
Table I. Primers used for quantitative polymerase chain reaction analysis.

Primers

Gene

Forward (5'-3')

Reverse (5'-3')

PCR products (bp)

$\begin{array}{ll}\beta \text {-actin } & \text { AGAAAATCTGGCACCACACC } \\ \text { Osteocalcin } & \text { GGCGCTACCTGTATCAATGG } \\ R U N X 2 & \text { CACCATGTCAGCAAAACTTCTT } \\ \text { Osterix } & \text { TGCTTGAGGAGGAAGTTCAC }\end{array}$

GGGGTGTTGAAGGTCTAAA

139

TCAGCCAACTCGTCACAGTC

106

TCACGTCGCTCATTTTGC

96

Osterix

AGGTCACTGCCCACAGAGTA

148

$R U N X 2$, runt-related transcription factor 2; bp, base pairs.
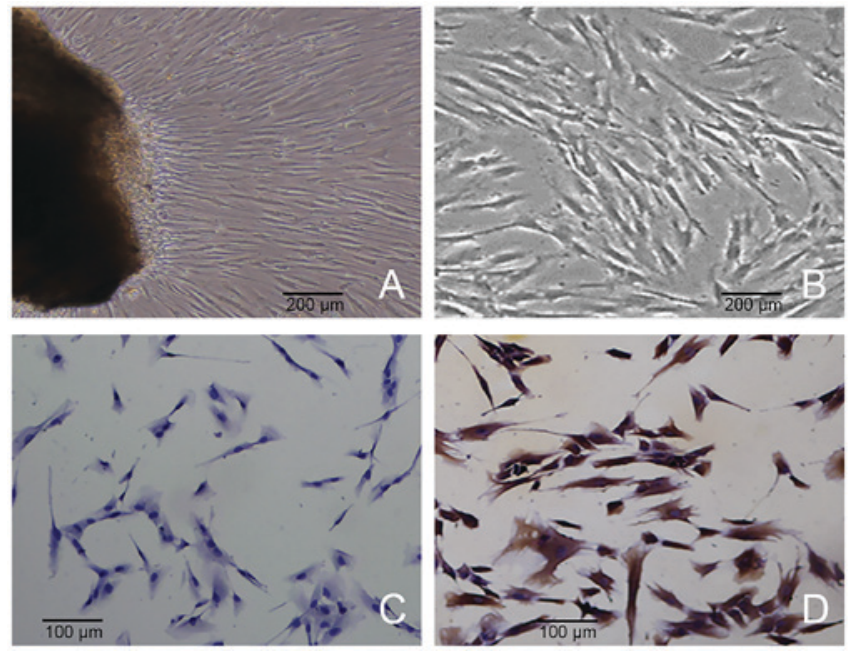

Figure 1. Identification and characterization of primary hPDLCs. (A) Primary cells grew out from the tissue explants and (B) were spindle shaped. Scale bar, $200 \mu \mathrm{m}$. (C) Immunocytochemistry staining showed that cells were vimentin positive and (D) cytokeratin negative. Scale bar, $100 \mu \mathrm{m}$. hPDLCs, human periodontal ligament cells.

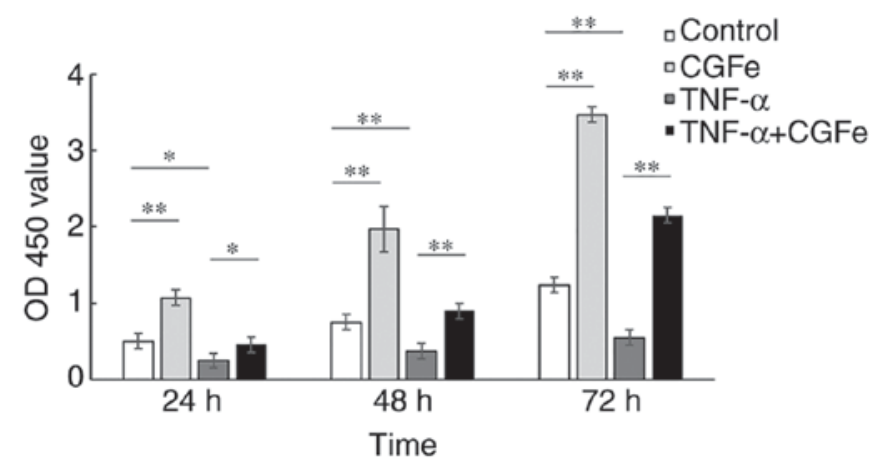

Figure 2. CGFe increases the proliferation of hPDLCs in vitro. The proliferation activity of hPDLCs was promoted by CGFe, but inhibited by TNF- $\alpha$. CGFe mitigated the effect of TNF- $\alpha .{ }^{~} \mathrm{P}<0.05,{ }^{, *} \mathrm{P}<0.01$. CGFe, concentrated growth factor exudate; hPDLCs, human periodontal ligament cells; TNF- $\alpha$, tumor necrosis factor- $\alpha$; OD, optical density.

CGFe increases hPDLC proliferation. The proliferation of hPDLCs was measured with CCK-8 assays (Fig. 2). After 24, 48 and $72 \mathrm{~h}, \mathrm{CGFe}$ significantly enhanced the proliferation of hPDLCs compared with the control group $(\mathrm{P}<0.01)$, while TNF- $\alpha$ markedly inhibited the proliferation of hPDLCs (for

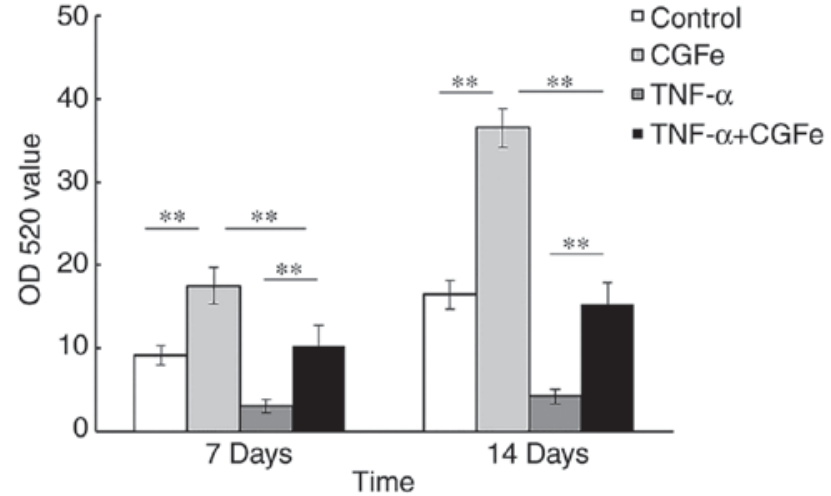

Figure 3. CGFe increases ALP activity in hPDLCs. After 7 or 14 days of culture, the ALP activity in the CGFe group was significantly higher than that of the control group. Compared with the control group, the ALP activity in the TNF- $\alpha$ group was decreased. ${ }^{* *} \mathrm{P}<0.01$. CGFe, concentrated growth factor exudate; hPDLCs, human periodontal ligament cells; TNF- $\alpha$, tumor necrosis factor- $\alpha$; ALP, alkaline phosphatase; OD, optical density.

TNF- $\alpha$ vs. control, $\mathrm{P}<0.05$ at 24 and $48 \mathrm{~h} ; \mathrm{P}<0.01$ at $72 \mathrm{~h}$ ). In addition, the proliferation rate of the CGFe group was significantly higher than that of the CGFe+TNF- $\alpha$ group, and the proliferation of the CGFe group exceeded the TNF- $\alpha$ group substantially (for TNF- $\alpha$ vs. CGFe+TNF- $\alpha, \mathrm{P}<0.05$ at $24 \mathrm{~h}$; $\mathrm{P}<0.01$ at 48 and $72 \mathrm{~h})$.

CGFe increases ALP activity. After 7 and 14 days of culture, the hPDLCs cultured in the CGFe group showed the highest levels of ALP activity compared with the other experimental groups (Fig. 3). Treatment with TNF- $\alpha$ significantly inhibited the ALP activity of hPDLCs, as shown by comparison of the TNF- $\alpha$ treatment group with the CGFe and TNF- $\alpha+\mathrm{CGFe}$ groups, and the difference between these groups was significant $(\mathrm{P}<0.01)$ at both time-points. As expected, the ALP activity in all groups progressively increased over time.

Alizarin Red S staining and semi-quantification of mineralized matrix nodules. In order to detect the formation of mineralized matrix nodules, Alizarin Red S staining was performed (Fig. 4A-D). After 21 days of osteogenic induction, mineralized nodules were observed with an inverted phase-contrast microscope, and the number of nodules was notably higher in the CGFe group (Fig. 4C), compared with the control (Fig. 4A) or CGFe+TNF- $\alpha$ (Fig. 4D) groups. The TNF- $\alpha$ group (Fig. 4B) formed the fewest mineralized nodules. 

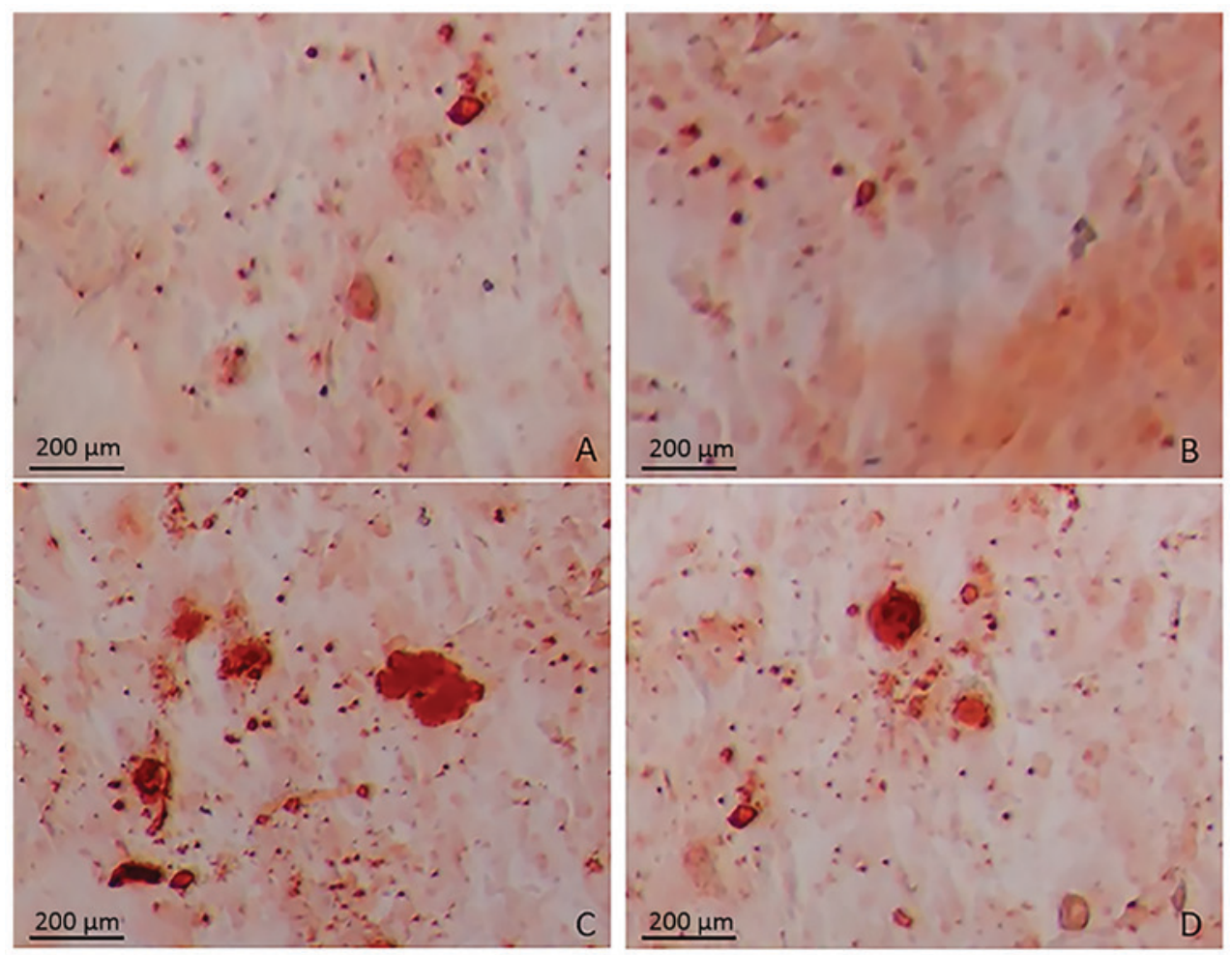

E

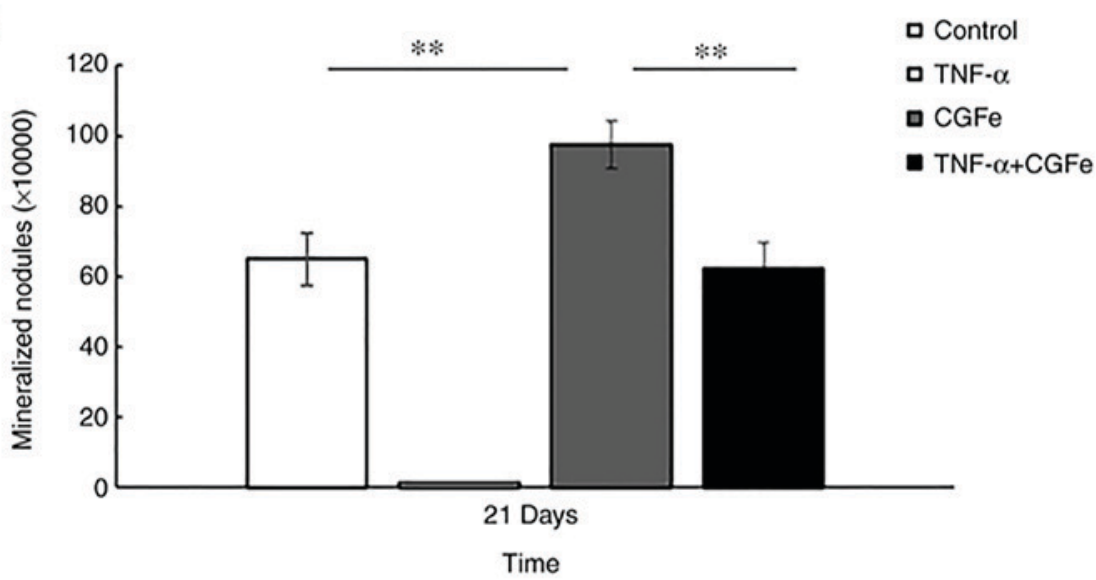

Figure 4. Alizarin Red S staining of hPDLCs. hDPLCs were cultured in (A) MM, (B) MM+TNF- $\alpha$, (C) MM+CGFe or (D) MM+CGFe+TNF- $\alpha$ (scale bar=200 $\mu \mathrm{m}$ ). After 21 days of osteogenic induction, the number of mineralized nodules in the MM+CGFe group was much greater than those in the other three groups. (E) Mineralized nodule number was quantified after 14 days of osteogenic induction. The number of mineralized nodules in the MM+CGFe group was increased compared with the MM+CGFe+TNF- $\alpha$ and control groups, and the control (MM) group formed more mineralized nodules than the TNF- $\alpha$ group. ${ }^{* *} \mathrm{P}<0.01$. MM, mineralization-inducing medium; CGFe, concentrated growth factor exudate; hPDLCs, human periodontal ligament cells; TNF- $\alpha$, tumor necrosis factor- $\alpha$.

The mineralized matrix nodules were quantified after 21 days of induction (Fig. 4E). The absorbance values at $570 \mathrm{~nm}$ revealed that extracellular calcium deposition in the CGFe group was significantly higher than the control or CGFe+TNF- $\alpha$ groups $(\mathrm{P}<0.01)$.

CGFe increases osteogenic-associated gene and protein expression. The gene expression of the RUNX2, OSX and $O C N$ was determined after 4, 7 and 14 days of osteogenic induction (Fig. 5). It was observed that on days 4,7 and 14 , the expression of $R U N X 2$ and $O S X$ in the TNF- $\alpha(10 \mathrm{ng} / \mathrm{ml})$ groups was decreased compared with the control group $(\mathrm{P}<0.01$; excluding OSX expression on day 7, $\mathrm{P}<0.05)$, and the expression of these two genes in the CGFe+TNF- $\alpha$ group showed no significant increase compared with the control group. By contrast, on days 4, 7, and 14, expression of the RUNX2 and OSX genes in the CGFe group was significantly increased, compared with the control group $(\mathrm{P}<0.01$; Fig. $5 \mathrm{~A}$ and $\mathrm{B})$. The expression of the downstream $O C N$ gene was not significantly different between groups on day 4 . By day 7 , the CGFe group began to surpass the control group $(\mathrm{P}<0.01)$. After 14 days of culture, $O C N$ expression in the TNF- $\alpha$ group was lower than that of the control group $(\mathrm{P}<0.01$; Fig. 5C).

Western blotting was performed to examine the effects of CGFe on hPDLC differentiation. hPDLCs were cultured in each medium for 14 days. As shown in Fig. 6A, compared 

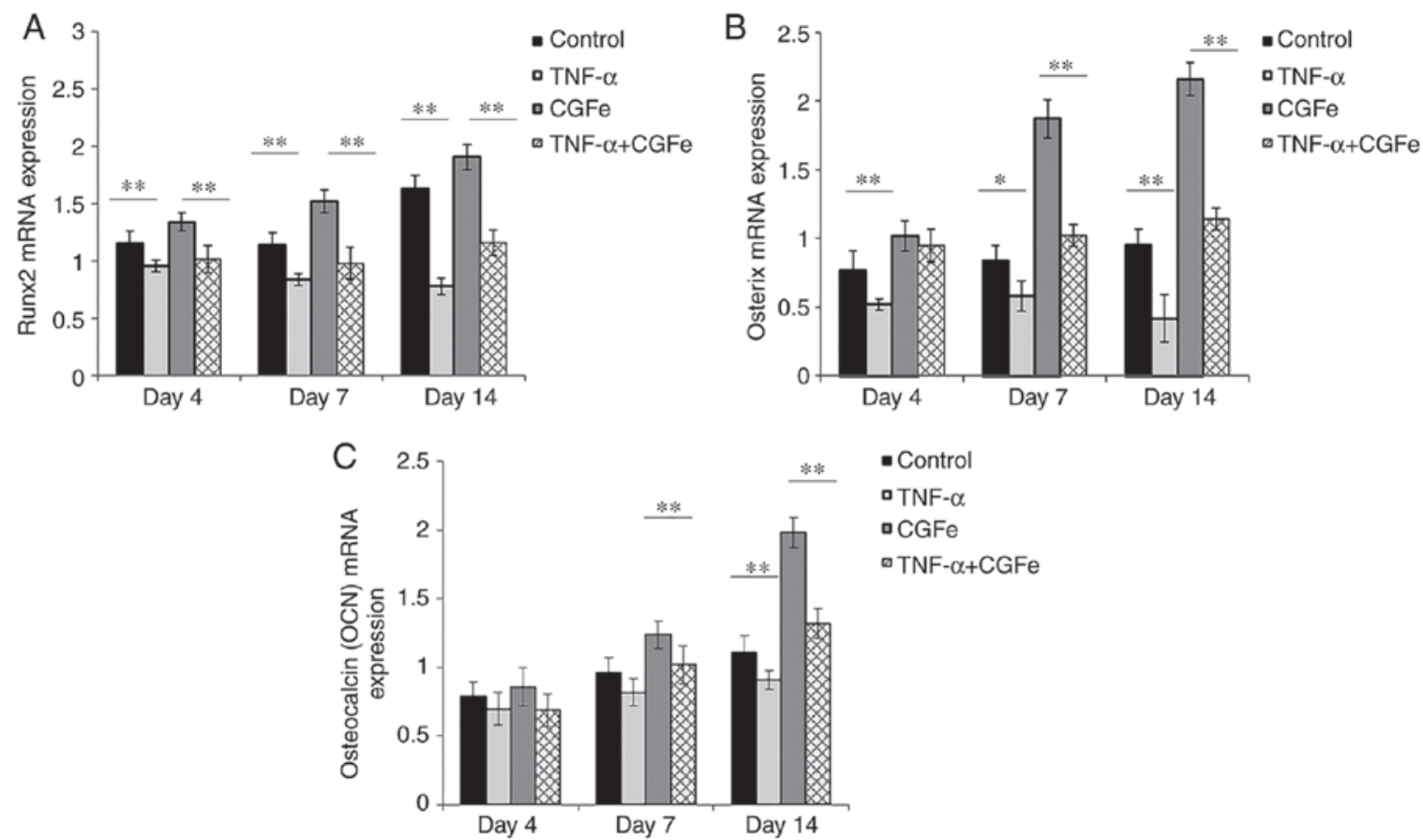

- Control

-TNF- $\alpha$

-CGFe

-TNF- $\alpha+$ CGFe

Figure 5. CGFe increases the expression of osteogenic-associated genes. Following osteogenic induction for 7 or 14 days, the expression of (A) RUNX2, (B) $O S X$ and (C) $O C N$ was determined by reverse transcription-quantitative polymerase chain reaction. The expression of $R U N X 2$ and $O S X$ in the TNF- $\alpha$ groups was lower than that of the control group. On days 4, 7 and 14, the expression of RUNX2 and OSX in the CGFe groups was higher than the control group. $O C N$ gene expression was not significantly different between the experimental groups on day 4. By day 7, OCN expression in the CGFe group began to surpass the control group. After 14 days of culture, $O C N$ expression in the TNF- $\alpha$ was lower than that of the control group. ${ }^{* *} \mathrm{P}<0.01$. CGFe, concentrated growth factor exudate; TNF- $\alpha$, tumor necrosis factor- $\alpha$; RUNX2, runt-related transcription factor 2; OCN, osteocalcin; OSX, Osterix.

A

osx

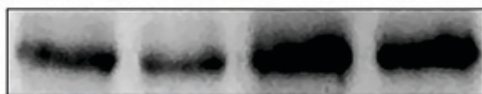

$45 \mathrm{kDa}$

Runx2

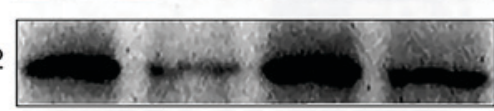

$55 \mathrm{kDa}$

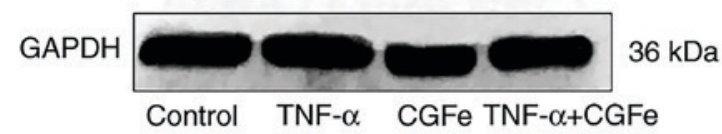

B

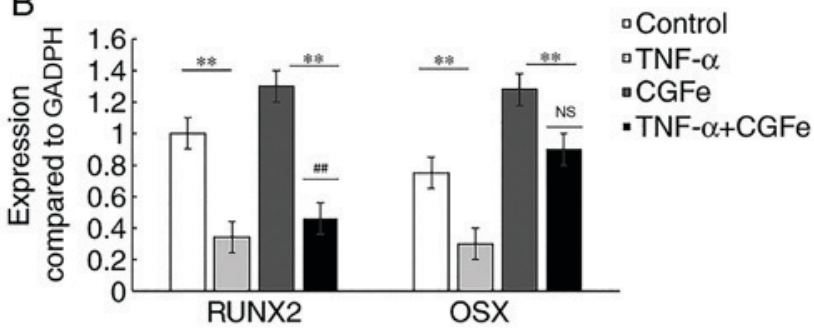

Figure 6. CGFe increases RUNX and OSX protein expression. (A) Western blot analysis of RUNX2 and OSX following treatment with various culture conditions for 14 days. GAPDH was used to monitor equal protein sample loading. (B) Quantitative analysis of RUNX2 and OSX protein expression. RUNX2 was upregulated in the CGFe group and downregulated in the CGFe + TNF- $\alpha$ and TNF- $\alpha$ groups. The protein level of OSX was upregulated in the CGFe+TNF- $\alpha$ group, but not significantly upregulated compared with the control group. ${ }^{* *} \mathrm{P}<0.01$; NS, CGFe+TNF- $\alpha$ group vs. control group ${ }^{\# \prime} \mathrm{P}<0.01, \mathrm{CGFe}+\mathrm{TNF}-\alpha$ group vs. control group. $\mathrm{CGFe}$, concentrated growth factor exudate; TNF- $\alpha$, tumor necrosis factor- $\alpha$; RUNX2, runt-related transcription factor 2; $O C N$, osteocalcin; $O S X$, Osterix.

with the control group, the protein expression of RUNX2 was upregulated in the CGFe group, and downregulated in the
CGFe+TNF- $\alpha$ and TNF- $\alpha$ groups $(\mathrm{P}<0.01$; Fig. 6B). A similar trend was observed for OSX expression. Although the protein level of OSX was upregulated in the CGFe+TNF- $\alpha$ group, there was no significant difference compared with the control group ( $\mathrm{P}>0.05$; Fig. 6B).

\section{Discussion}

CGFe can be obtained from the venous blood of patients and thus is convenient and economical to prepare. CGFe contains high concentrations of growth factors and provokes no immune reaction $(8-10,22,25)$. According to previous studies (4-8), the CGFe obtained from different patients in the present study contained different inventories of components, yet the major components were the same. These included epidermal growth factor, platelet-derived growth factor, transforming growth factor (TGF- $\beta$ ), vascular endothelium growth factor, insulin-like growth factor, bone morphogenetic protein (BMP), and fibroblast growth factor (4-8). In the last decade, CGFe has been widely used in the reconstruction of bone tissue in the surgical field $(8,9)$. These growth factors have functions in accelerating the revascularization of injured tissues and inducing the migration, proliferation, and differentiation of fibroblasts and osteoblasts $(8-10,25)$.

TNF- $\alpha$ is a common and crucial inflammatory factor in the development of periodontal inflammation and alveolar bone resorption (20). It has been established that TNF- $\alpha$ significantly inhibits the proliferation of hPDLCs and mesenchymal stem cells $(5,26,27)$. In addition, activation of the nuclear factor (NF)- $\mathrm{kB}$ signaling pathway inhibits the expression of $R U N X 2$, which is a downstream transcription factor induced 
by the exogenous BMP2-mothers against decapentaplegic homolog (Smad) signaling pathway controlling osteoblast differentiation, thus inhibiting the formation of bone (28).

In the present study, TNF- $\alpha(10 \mathrm{ng} / \mathrm{ml})$ was used to construct an inflammatory microenvironment. It was demonstrated TNF- $\alpha$ reduced ALP activity, mineralization ability and the expression of RUNX2 and OSX, compared with the control group. This result was concordant with the findings of Yang et al (18), which revealed that the bone formation efficiency of gingival mesenchymal stem cells and periodontal ligament stem cells decreased under TNF- $\alpha$-induced inflammatory conditions.

Previous studies have shown that RUNX2 and OSX are two essential transcription factors in the osteogenic pathway $(29,30)$. In particular, RUNX2 regulates cell osteogenic differentiation and serves a central role in multiple osteogenic signaling pathways (29). OSX is an osteogenic-specific transcription factor with a zinc finger structure. A previous study revealed that no bone formation occurs in mice lacking the $O S X$ gene (30). The level of ALP activity reflects the tendency of cells to transform in the osteogenic direction midway during the osteoproductive process, while OCN is a late marker of osteogenesis. OCN is the most abundant non-procollagen protein in bone tissue and promotes osteogenic differentiation by combining with minerals $(31,32)$. Positive Alizarin Red S staining of the mineralized nodules formed indicates the expression of OCN and osteogenesis at a later stage $(31,32)$.

In the present study, CGFe was added to cell cultures to observe the osteogenic efficiency of hPDLCs, by detecting the expression of RUNX2, OSX and OCN genes, as well as ALP activity and mineralized nodule formation in each group of hPDLCs. The formation of mineralized nodules was used to determine the osteogenic capacity of each group of hPDLCs. It was observed that even in the presence of inflammatory cytokine stimulation, hPDLCs cultured with CGFe still exhibited osteogenic differentiation and mineralization ability. By comparing the assay and imaging results of the CGFe group with the CGFe+TNF- $\alpha$ group, it was demonstrated that ALP activity in the CGFe group was stronger than that of the CGFe+TNF- $\alpha$ group, and the formation of mineralized nodules was notable in both groups, although the CGFe group was stained more prominently. In addition, ALP activity in the CGFe+TNF- $\alpha$ group was significantly higher than the TNF- $\alpha$ group, and the CGFe+TNF- $\alpha$ group had deep mineralized staining with a large area and high density, while no mineralized nodule formation was observed in the TNF- $\alpha$ group.

The western blotting results of the present study revealed that RUNX2 and OSX expression was higher in the CGFe group, compared with the $\mathrm{CGFe}+\mathrm{TNF}-\alpha$ group, indicating that $\mathrm{TNF}-\alpha$ may play an inhibitory role upstream of protein translation. By contrast, growth factors contained in the $\mathrm{CGFe}$ may antagonize the inhibitory effect of TNF- $\alpha$ on cell proliferation. For example, TGF- $\beta 1$ induces the phosphorylation of Smad2/Smad3, which upregulates $R U N X 2$ transcription (29), thereby impeding TNF- $\alpha$ activation of the NF- $\kappa \mathrm{B}$ signaling pathway, which would result in RUNX2 inhibition.

In conclusion, CGFe not only has an osteogenic effect on hPDLCs in a normal culture, but also promotes hPDLC osteogenesis in a TNF- $\alpha$-induced inflammatory microenvironment. In the present work, a single inflammatory factor, $\mathrm{TNF}-\alpha$, was used to mimic the microenvironment of periodontal disease, and this imposed certain limitations. The enhancing effect of CGFe on osteogenic differentiation of hPDLCs stimulated by other inflammatory factors will be examined in follow-up experiments. To study the effects of CGFe in the treatment of alveolar bone defects, in vivo should be performed. In addition, the exact mechanism of how CGFe enhanced the proliferation and osteogenic differentiation of hPDLCs should be determined.

\section{Acknowledgements}

Not applicable.

\section{Funding}

This work was supported by a grant from the People's Hospital of Longhua (Shenzhen, China) and the Health and Family Planning Commission of Shenzhen Municipality (grant no. SZFZ2018035).

\section{Availability of data and materials}

The datasets used and/or analyzed during the current study are available from the corresponding author on reasonable request.

\section{Authors' contributions}

XL, HY and BW conceived and designed the study. XL, YZ, and HL performed cell culture, immunostaining and proliferation analysis. XL and YZ performed the experimental procedures of osteogenic differentiation induction, RT-qPCR, and western blotting. $\mathrm{HY}, \mathrm{ZZ}$ and $\mathrm{ZY}$ provided reagents and interpreted the data. XL, HY and BW performed data analysis and wrote the manuscript. All authors read and approved the final manuscript.

\section{Ethics approval and consent to participate}

This study was approved by the Ethics Committee of the Jilin University Health Science Center (Changchun, China). Written consent was obtained prior to experimentation.

\section{Patient consent for publication}

Not applicable.

\section{Competing interests}

The authors declare that they have no competing interests.

\section{References}

1. Alsaadi G, Quirynen M, Komárek A and Van Steenberghe D: Impact of local and systemic factors on the incidence of oral implant failures, up to abutment connection. J Clin Periodontol 34: 610-617, 2010.

2. Del Fabbro M, Boggian C and Taschieri S: Immediate implant placement into fresh extraction sites with chronic periapical pathologic features combined with plasma rich in growth factors: Preliminary results of single-cohort study. J Oral Maxillofac Surg 67: 2476-2484, 2009. 
3. Qiao J, Duan J, Zhang Y, Chu Y and Sun C: The effect of concentrated growth factors in the treatment of periodontal intrabony defects. Future Sci OA 2: FS136, 2016.

4. Jang SJ, Kim JD and Cha SS: Platelet-rich plasma (PRP) injections as an effective treatment for early osteoarthritis. Eur J Orthop Surg Traumatol 23: 573-580, 2013.

5. He L, Lin Y, Hu X, Zhang Y and Wu H: A comparative study of platelet-rich fibrin (PRF) and platelet-rich plasma (PRP) on the effect of proliferation and differentiation of rat osteoblasts in vitro. Oral Surg Oral Med Oral Pathol Oral Radiol Endod 108 707-713, 2009.

6. Suba Z, Takács D, Gyulai-Gaál S and Kovács K: Facilitation of beta-tricalcium phosphate-induced alveolar bone regeneration by platelet rich plasma in beagle dogs: A histologic and histomorphometric study. Int J Oral Maxillofac Implants 19: 832-838, 2004.

7. Fennis JP, Stoelinga PJ and Jansen JA: Mandibular reconstruction: A histologic and histomorphomrtric study on the use of autogenous scaffolds, particulate cortico-cancellous bone grafts and platelet rich plasma in goats. Int J Oral Maxillofac Surg 33: 48-55, 2004.

8. Kim TH, Kim SH, Sándor GK and Kim YD: Comparison of platelet-rich plasma (PRP), platelet-rich fibrin (PRF), and concentrated growth factor (CGF) in rabbit-skull defect healing. Arch Oral Biol 59: 550-558, 2014

9. Park HC, Kim SG, Oh JS, You JS, Kim JS, Lim SC, Jeong MA Kim JS, Jung C, Kwon YS and Ji H: Early bone formation at a femur defect using CGF and PRF grafts in adult dogs: A comparative study. Implant Dent 25: 387-393, 2016.

10. Rodella LF, Favero G, Boninsegna R, Buffoli B, Labanca M, Scarì G, Sacco L, Batani T and Rezzani R: Growth factors, CD34 positive cells, and fibrin network analysis in concentrated growth factors fraction. Microsc Res Tech 74: 772-777, 2011.

11. Li XJ, Yang HX, Zhang ZJ, Yan ZH, Lv HL, Zhang Y and Wu B: Platelet-rich fibrin exudate promotes the proliferation and osteogenic differentiation of human periodontal ligament cells in vitro. Mol Med Rep 18: 4477-4485, 2018.

12. Dohan DM, Choukroun J, Diss A, Dohan SL, Dohan AJ, Mouhyi J and Gogly B: Platelet-rich fibrin (PRF): A second generation platelet concentrate. Part I: Technological concepts and evolution. Oral Surg Oral Med Oral Pathol Oral Radiol Endod 101: e37-e44, 2006.

13. Tavassoli-Hojiati S, Sattari M, Ghasemi T, Ahmadi R and Mashayekhi A: Effect of platelet-rich plasma concentrations on the proliferation of periodontal cells: An in vitro study. Eur J Dent 10: 469-474, 2016.

14. Dohan DM, Choukroun J, Diss A, Dohan SL, Dohan AJ, Mouhyi J and Gogly B: Platelet-rich fibrin (PRF): A second-generation platelet concentrate. Part III: Leucocyte activation: A new feature for platelet concentrates? Oral Surg Oral Med Oral Pathol Oral Radiol Endod 101: e51-e55, 2006.

15. Li B and Wang Y: Contour changes in human alveolar bone following tooth extraction of the maxillary central incisor J Zhejiang Univ Sci B 15: 1064-1071, 2014.

16. Lacey DC, Simmons PJ, Graves SE and Hamilton JA: Proinflammatory cytokines inhibit osteogenic differentiation from stem cells: Implications for bone repair during inflammation. Osteoarthritis Cartilage 17: 735-742, 2009.

17. Yu B and Wang Z: Effect of concentrated growth factors on beagle periodontal ligament stem cells in vitro. Mol Med Rep 9: $235-242,2014$
18. Yang $\mathrm{H}$, Gao LN, An Y, Hu CH, Jin F, Zhou J, Jin $\mathrm{Y}$ and Chen FM: Comparison of mesenchymal stem cells derived from gingival tissue and periodontal ligament in different incubation conditions. Biomaterials 34: 7033-7047, 2013.

19. Górska R, Gregorek H, Kowalski J, Laskus-Perendyk A, Syczewska M and Madaliński K: Relationship between clinical parameters and cytokine profiles in inflamed gingival tissue and serum samples from patients with chronic periodontitis. J Clin Periodontal 30: 1046-1052, 2003

20. Li W, Yu B, Li M, Sun D, Hu Y, Zhao M, Cui CB and Hou S: NEMO-binding domain peptide promotes osteoblast differentiation impaired by tumor necrosis factor alpha. Biochem Biophys Res Commun 391: 1228-1233, 2010.

21. Sacco L: Lecture: International Academy of Implant Prosthesis and Osteoconnection. Lecture 12: 4, 2006.

22. Sohn DS, Heo JU, Kwak DH, Kim DE, Kim JM, Moon JW, Lee JH and Park IS: Bone regeneration in the maxillary sinus using an autologous fibrin-rich block with concentrated growth factors alone. Implant Dent 20: 389-395, 2011.

23. Yamaguchi A: Application of BMP to bone repair. Clin Calcium 17: 263-369, 2007 (In Japanese).

24. Livak KJ and Schmittgen TD: Analysis of relative gene expression data using real-time quantitative PCR and the 2(-Delta Delta C(T)) method. Methods 25: 402-408, 2001

25. Honda H, Tamai N, Naka N, Yoshikawa H and Myoui A: Bone tissue engineering with bone marrow-derived stromal cells integrated with concentrated growth factor in Rattus norvegicus calvaria defect model. J Artif Organs 16: 305-315, 2013.

26. Choukroun J, Diss A, Simonpieri A, Girard MO, Schoeffler C, Dohan SL, Dohan AJ, Mouhyi J and Dohan DM: Platelet-rich fibrin (PRF): A second-generation platelet concentrate. Part V: Histologic evaluations of PRF effects on bone allograft maturation in sinus lift. Oral Surg Oral Med Oral Pathol Oral Radiol Endod 101: 299-303, 2006.

27. Kang YH, Jeon SH, Park JY, Chung JH, Choung YH, Choung HW, Kim ES and Choung PH: Platelet-rich fibrin is a Bioscaffold and reservoir of growth factors for tissue regeneration. Tissue Eng Part A 17: 349-359, 2011.

28. Clipet F, Tricot S, Alno N, Massot M, Solhi H, Cathelineau G, Perez F, De Mello G and Pellen-Mussi P: In vitro effects of Choukroun's platelet-rich fibrin conditioned medium on 3 different cell lines implicated in dental implantology. Implant Dent 21: 51-56, 2012.

29. Franceschi RT and Xiao G: Regulation of the osteoblast-specific transcription factor, Runx2: Responsiveness to multiple signal transduction pathways. J Cell Biochem 88: 446-454, 2003.

30. Nakashima K,Zhou X,Kunkel G,Zhang Z, Deng JM,Behringer RR and de Crombrugghe B: The novel zinc finger-containing transcription factor osterix is required for osteoblast differentiation and bone formation. Cell 108: 17-29, 2002

31. Piek E, Ju WJ, Heeyer J, Escalante-Alcalde D, Stewart CL, Weinstein M, Deng C, Kucherlapati R, Bottinger EP and Roberts AB: Functional characterization of transforming growth factor beta signaling in Smad2-and Smad3-deficient fibroblasts. J Biol Chem 276: 19945-19953, 2001.

32. Glowacki J, Rey C, Glimcher MJ, Cox KA and Lian J: A role for osteocalcin in osteoclast differentiation. J Cell Biochem 45: 292-302, 1991

(c) (i) () $($ This work is licensed under a Creative Commons Attribution-NonCommercial-NoDerivatives 4.0 International (CC BY-NC-ND 4.0) License. 